Research Article

\title{
Fault Diagnosis and Identification of Power Capacitor Based on Edge Cloud Computing and Deep Learning
}

\author{
Xiangbing Zhao, Xulong Zhang $(\mathbb{D}$, and Peihua Ren \\ School of Computer and Network Engineering, Shanxi Datong University, Datong 037009, China \\ Correspondence should be addressed to Xulong Zhang; zxllongge2018@sxdtdx.edu.cn
}

Received 4 July 2020; Revised 25 July 2020; Accepted 29 July 2020; Published 26 August 2020

Academic Editor: Sang-Bing Tsai

Copyright (c) 2020 Xiangbing Zhao et al. This is an open access article distributed under the Creative Commons Attribution License, which permits unrestricted use, distribution, and reproduction in any medium, provided the original work is properly cited.

\begin{abstract}
Nowadays, power electronic technology is widely affecting people's daily work and life. However, there are still many problems in the current power supply research. When the fault information of power transformer is not complete or there is some ambiguity or even the information is lost, it will largely lead to the conclusion and correct conclusion of fault diagnosis. In this case, the fuzzy theory is applied to the fault diagnosis of shunt capacitor, and the fuzzy fault diagnosis system of shunt capacitor is studied. At the same time, a map-based fault diagnosis system is proposed. In this paper, the cloud computing technology is introduced into the deep learning and compared with SVM and DBN algorithm. The research results of this paper show that the accuracy of fuzzy diagnosis results is $94 \%, 84 \%, 90 \%, 80 \%, 83 \%$, and $70 \%$, respectively, which shows that the model diagnosis reliability is relatively high. Among the three algorithms, MR-DBN overall detection rate is higher and the time-consuming is lower than the other two methods. The diagnostic accuracy and misjudgment rate of DBN are as follows: $96.33 \%$ and 3.90\%. The diagnosis accuracy and misjudgment rate of SVM are as follows: $96.40 \%$ and 3.83\%. The diagnostic accuracy and misjudgment rate of MR-DBN are, respectively, $99.52 \%$ and $0.57 \%$. Compared with the other two methods, MR-DBN has the highest diagnostic accuracy and the lowest error rate, which to a large extent indicates that MR-DBN algorithm has higher diagnostic accuracy and has greater advantages and reliability in power supply diagnosis and identification. It not only improves the accuracy of power capacitor fault diagnosis and identification but also provides a new method for the application of power capacitor fault research and development.
\end{abstract}

\section{Introduction}

With the rapid development of science and technology, fault diagnosis has been paid more and more attention. Reactive power compensation and voltage support have become a decisive factor for the safe and stable operation of power grid. Power capacitor is the basic component of reactive power compensation device in power system. By changing the reactive flow in power system, the power can be greatly improved. The voltage level in the power system can reduce the related power loss and improve the related state performance of the power system; therefore, the normal operation of the power capacitor is related to the stability and economy of the power system operation.

At present, based on the continuous and rapid growth of power demand and the rapid development of smart grid, the relevant capacity of power system network is also becoming larger and larger and gradually developing towards the direction of high voltage and ultrahigh voltage; however, with the continuous development of power system, the impact and loss caused by the relevant faults of power system equipment are more and more; therefore, high precision is proposed. The method of power capacitor fault diagnosis and identification based on degree is very important.

Wang et al. put forward a kind of capacitor dielectric loss factor identification algorithm based on deep learning, trained a feedforward multilayer artificial neural network with online sampling period, identified the dielectric loss angle from the new monitoring data with resolution of $0.001 \%$, put forward the calculation method of dielectric loss factor identification signal $\mathrm{D} \delta(\mathrm{T})$, and verified that the 
amplitude of $\mathrm{D} \delta(\mathrm{T})$ is dielectric loss angle, and the waveform shape includes the interference of the monitoring device. Although the dielectric loss factor identification algorithm has accuracy, it still lacks a certain economy [1]. Chen et al. proposed a correlation model for fault diagnosis and prediction of thermal power units based on deep learning and multimedia system, which greatly improved the balance between power systems. The research results of Chen et al. show that this method is reliable. Although this method has stability and reliability, it still lacks certain practicability [2]. Based on the deep learning algorithm, Jian et al. constructed a layered automatic coding network. By introducing sparse constraints to compress and reduce the input data, the network can accurately extract the fault characteristics of the input data and improve the fault identification ability of the network by introducing random noise. Although the stack automatic coding network has accuracy, it lacks certain stability [3].

In this paper, the fuzzy theory is applied to the fault diagnosis of shunt capacitor, and on this basis, a fuzzy fault diagnosis system of shunt capacitor is studied, which can accurately diagnose what kind of fault happened to capacitor and its related severity to a great extent; at the same time, a distributed DBN based on Map Reduce is used in this paper. It introduces deep learning and this is compared with SVM and DBN, which highlights the advantages and reliability of MR-DBN algorithm, provides related services for fault diagnosis and identification of capacitors, improves the accuracy of fault diagnosis and identification of capacitors, and provides some theoretical experience for the research and application of capacitor faults.

\section{Edge Calculation and Power Capacitor}

\subsection{Edge Computing}

2.1.1. Concept of Edge Computing. Edge computing belongs to a distributed open platform, which integrates core functions such as network, computing, storage, and application on the edge of the network. It provides relevant edge intelligence services nearby, so as to meet the requirements of agile connection, real-time business, data optimization, application intelligence, security, and privacy protection to a large extent. Edge computing can not only connect the related physical and digital worlds but also be used in intelligent assets, intelligent gateways, and intelligent systems, as well as intelligent services that provide some effective information [4].

2.1.2. Edge Computing Advantages. There will be an open interface about the model in each layer of the edge computing reference architecture, so as to fully open the architecture. The related reference architecture of the edge computing can realize the whole business process and the whole life cycle intelligent service interface through vertical management services and security services to a large extent; compared with the traditional cloud computing model, the edge computing model can play an important role in the network to a large extent and can better support the application of the Internet of things. Its advantages mainly lie in the following two aspects:

(1) Relieve the Pressure of Network Bandwidth and Data Center. The typical feature of Internet of things data is high redundancy, most of which are temporary data. Edge computing can make scientific and effective use of this function to process a large number of temporary data at the edge of the network, so as to reduce its adverse impact on the network bandwidth and data center [5].

(2) High Data Security. In the relevant mode of cloud computing, all the data are concentrated in the data center, which makes it difficult for users to control the access and use of relevant data. However, edge computing can store and use the data in the network edge device closer to the user, thus greatly improving the security of data [6].

\subsection{Power Capacitor}

2.2.1. Power Capacitor. Power capacitor refers to a kind of nondynamic reactive power compensation device. Its main function is to provide reactive power for the power system, so as to improve the relevant power factor to a large extent. Using local reactive power compensation can not only greatly reduce the transmission current of transmission line but also greatly reduce the related loss of line energy and improve the equipment benefit utilization which plays an important role [7]. In addition, capacitors play a very important role in improving the power quality of power system, which is an important means to ensure the economic and safe operation of power system. Its safe operation and fault handling are very important. In the long-term operation, due to the influence of operation environment, human factors, design problems, and other related factors, the phenomenon of fault is common, which to a large extent has a bad impact on the safe operation of the power system.

2.2.2. Structural Advantage. Modern high-voltage shunt capacitor elements mainly use oil-immersed polypropylene film as the medium between electrodes and aluminum foil as the plate. One end of the plate extends out of the medium and the other end folds and sinks into the medium [8]; the structural advantages of this plate are as follows:

(1) The electric field distribution at the edge of the plate is improved

(2) The PD performance is improved obviously

(3) The related resistance and loss of the electrode plate are greatly reduced, the speed of temperature rise is also reduced, and the thermal stability is also improved

(4) The protruding plate can be used to connect components, thus greatly simplifying the structure and manufacturing process and saving related labor time [9] 
In many related engineering practices, it is necessary to make a detailed analysis of the electric field distribution law of the weakest part of the board edge insulation and the relative strength between the largest electric fields, so as to ensure the safety and reliability to a large extent. In general, it is enough to make a general analysis and understanding of the electric field distribution law of the rest parts, and it is not necessary to accurately calculate the board. It is not necessary to accurately calculate the electric field intensity of each point in the edge area of the plate so that the simplified calculation method can be carried out and the calculation time can be saved [10].

2.2.3. Related Relationship. With the continuous increase of the average electric field strength of the capacitor, the maximum electric field strength at the edge of the board increases synchronously. The voltage of the capacitor unit is the product of the number of element strings and the voltage of the element. Increasing the element voltage can reduce the number of element strings to a large extent, which makes the relevant structure and manufacturing of the capacitor more and more simplified without increasing the average field. Under the premise of strong, the related consumption of aluminum foil can be saved, and the related cost can be reduced to a large extent [11]. However, the continuous increase of the working voltage of the module will increase the film thickness between the two poles, so as to increase the correlation coefficient of electric field distortion and the maximum field strength at the edge of the plate [12].

It can be said that the maximum field strength at the edge of the capacitor plate is one of the most fundamental and influential factors restricting the development of power capacitor technology. Generally, the choice of the working field strength of the capacitor is concerned, that is, the average field strength. On the premise of ensuring reliability, the higher the average field strength is, the lower the volume and weight cost of the corresponding capacitor is and the higher the level of the capacitor is; conversely, in order to ensure the safety and reliability of the capacitor, there is a method that requires strict control of the average field strength not greater than a certain value. This method only focuses on the impact of the average field strength and ignores the impact of the maximum field strength at the edge of the plate [13].

\subsection{Fault Diagnosis and Maintenance of Power Capacitor}

2.3.1. Power Capacitor Leakage Oil. The leakage of oil is mainly caused by loose seal or insecure seal. The capacitor is a completely sealed equipment. If the seal is not tight, air, moisture, and impurities may enter the oil tank, which will cause certain damage to the insulation layer, resulting in serious damage. Therefore, the capacitor is not allowed to leak oil. In fact, the leaking parts are mainly at the welds and casings of the oil tank, which indicates that the welding process of these parts is poor, and the manufacturer has not carried out strict requirements for the sealing test, instead of conducting the leakage test one by one. According to the general standard, it should be heated to $75^{\circ} \mathrm{C}$ and kept for 2 hours to carry out the test. When purchasing, the manufacturer should be strictly required to carry out the test. In fact, the leaking part of the casing pipe is mainly the root, cover, bolt, and other welded joints. The causes of the leakage mainly include processing technology problems, structural design problems, and human factors. The mechanical strength of the welding used for the bolt and cover is very poor. If the tightening force is slightly large, the screw will be removed for welding. Therefore, when the temperature changes, the bolt will be applied with a certain degree of pressure. It is easy to open the screw welded joint; in addition, the sleeve is lifted directly during the transportation process, so careless treatment will also make the weld crack. In view of the above reasons, corresponding measures should be taken to strengthen the management of production enterprises and operation and maintenance personnel, so as to solve the relevant leakage problems to a certain extent [14].

2.3.2. Poor Insulation. This kind of phenomenon is found in the preventive test; basically, there are two situations as follows:

(1) In the long-term heating and voltage life test, the change of capacitance value is very small. If the capacitance value suddenly increases, it can only be regarded as the breakdown and short circuit of some capacitor elements. Because the capacitor is composed of multiple components in series, the capacitance will increase only when the number of series segments is reduced, and the capacitance value will decrease if some elements are disconnected.

(2) The second part of the poorly insulated capacitor is that the dielectric loss angle exceeds a certain range, which will slightly increase the dielectric loss angle of the capacitor during long-term operation, and the multiple increase is abnormal, because only when partial discharge and partial overheating occur, the dielectric loss angle will exceed a certain range, so it can only be replaced. The insulation strength of the welding electrode to the oil tank is usually very high, but due to the defects in the welding process, such that the insulating cardboard between the parts and the oil tank is burnt out, the wire is not insulated, the oil is insufficient, the short tail sleeve is used, the insulation distance is insufficient, and the quality of the porcelain sleeve is poor, during the relevant test process, the discharge and burst of the sleeve may occur. Therefore, patrol inspection should be strengthened, hidden dangers should be found in time, and corresponding treatment should be carried out [15].

(3) Capacitor case bulge: in case of large expansion of the capacitor shell, it is caused by excessive internal pressure. The internal pressure is too high due to the gas produced by the internal free medium or the gas produced by breakdown, discharge, and other 
phenomena. The generated gas will increase the internal pressure of the capacitor and cause the phenomenon of shell expansion [16]. When the above phenomenon occurs, it should be handled in time.

(4) Capacitor explosion: when a capacitor breaks down or discharges between poles due to a sharp increase of internal energy, the capacitor will explode. This usually happens in a capacitor without internal fuse. Many lowvoltage capacitors are equipped with protection fuses, so low-voltage capacitors rarely explode. The short-circuit breakdown characteristics of paper film capacitors and full film capacitors are different, so they are released by the authority after electricity, and the insulating paper will be carbonized under high temperature, thus forming the capacitor element of full film and paper film composite dielectric. Because the separation of carbonized paper will keep discharging for a period of time, a large amount of gas will be generated at this time. If there is no fuse protection, the oil tank will break. After discharging, the film of full film capacitor will be affected by high temperature, and melting will make two-electrode short-circuit contact without arc discharge or gas explosion, so full film capacitor shall be used for explosion proof [17].

(5) Capacitor heating: when there are harmonics in the system, the harmonic current will damage the capacitor to a certain extent, which will aggravate the related loss of the insulation medium of the capacitor, so it is easy to make the insulation layer age rapidly; sometimes, it may bring the related risk of thermal breakdown, which will have a bad impact on the safe operation of the capacitor to a large extent, in the case of harmonics, the electrode of the capacitor. The voltage between them may reach a large value, which may cause the risk of internal discharge of the capacitor and threaten the insulation of the power container, which may easily cause the capacitor to heat [18].

(6) Environmental temperature problems: there is a certain range of temperature around the capacitor, which should not be too high or too low. If the ambient temperature exceeds a certain safety range, the heat generated by the capacitor during operation cannot be eliminated; if the ambient temperature is lower than a certain range, the oil in the capacitor may freeze and be easily broken by electricity [19].

(7) Maintenance of power capacitor: the power capacitor bank in operation shall be subject to routine patrol inspection, relevant maintenance and repair, and regular power outage inspection [20].

(1) The capacitor shall be equipped with relevant personnel on duty, and the operation of the equipment shall be recorded.

(2) The working capacitor bank shall be subject to certain appearance inspection every day according to the regulations. If the expansion of the shell is noticed, it shall be stopped to avoid damage.

(3) Check the load of each phase of capacitor bank with ammeter.

(4) When the capacitor bank is put into use, the ambient temperature under normal conditions shall not be lower than $-4^{\circ} \mathrm{C}$, the ambient temperature during operation shall not be higher than $+40^{\circ} \mathrm{C}$, and the average ambient temperature during operation shall not be higher than $+30^{\circ} \mathrm{C}[21]$.

(5) After connecting the capacitors, it will cause the grid voltage to rise, especially in light load; in this case, some or all capacitors shall be disconnected from the grid [22].

(6) The surface of capacitor and supporting insulator shall be cleaned regularly to ensure that no damage or discharge trace is found. At the same time, the shell of capacitor shall also be cleaned to ensure that there is no deformation and oil leakage, and the capacitor and iron frame shall not be covered with dust and other pollutants.

(7) It is necessary to pay attention to the reliability of all contacts on the circuit connected to the capacitor bank (current bank, ground wire, circuit breaker, fuse, switch, etc.), as long as there is a contact fault in the circuit, even if the nut is loose, the capacitor may be damaged prematurely and the whole equipment will be damaged.

(8) If the capacitor needs to pass the withstand voltage test for a period of time, the relevant test shall be carried out according to the specified value.

\subsection{Common Troubleshooting and Preventive Measures}

(1) In case of fire such as discharge and explosion of capacitor, cut off the power supply first, and then put out the fire.

(2) When the corresponding circuit breaker of the capacitor trips, first discharge the capacitor completely, and then check the condition of the relevant equipment. If there is no abnormality in the inspection, it may be caused by the fluctuation of the grid voltage. If the operation is abnormal, it may be caused by the internal fault of the capacitor. Check and test each capacitor until the relevant cause of the fault is found [23].

(3) When the fuse is damaged, the capacitor shall be discharged completely first, then the fuse shall be replaced, and the corresponding equipment can be put into use only after no other abnormal phenomenon is found. If the trial operation is not successful, each capacitor shall be inspected and tested after power failure. 
(4) Control the working temperature correctly; do not work at a temperature higher than $60^{\circ} \mathrm{C}$. If the temperature rises, pay attention to ventilation. If it is not caused by ventilation problems, quickly find out the causes and deal with the abnormalities, and do not operate at high temperature for a long time [24].

(5) In the process of capacitor installation, the method of series reactor can prevent the harm of harmonic to a large extent and plays a crucial role in the overall operation [25].

\section{Deep Learning}

3.1. Deep Learning. Deep learning technology is the latest achievement in machine learning research. Deep learning model is a breakthrough of the original neural network model, which imitates the abstract reflection of human brain to objective things. The difference between the deep learning model and the neural network model is that the deep learning model can largely solve the overfitting problem when training the multilayer neural network. The depth model is composed of multilayer neural network. There is no mutual connection between nodes in the same layer of neural network. It uses greedy algorithm to train each layer of network. When the layer of network reaches the accuracy requirements, it begins to train the next layer. Each layer is an abstract expression of different sides of the original data. For example, an image is composed of many pixels, many pixels form part of the image, and some regional images form the whole image; for example, statements are composed of a single character, multiple characters form words, multiple words form sentences with meaning similarly, network data packets are composed of multiple binary bits, multiple binary bits form each protocol field, and protocol fields form a protocol.

Because the depth model is layer-by-layer learning, learning a higher-level representation of the previous layer, that is, the simulation of the previous layer in different dimensions, so given the sample data, the process of depth learning is the process of approaching the distribution or function of the sample data. The deep learning model is mainly composed of input-output layer and multiple hidden layers. The data is input from input layer and output from output layer through the transformation of each hidden layer. The purpose of training model is to make the information of output layer consistent with the total information of input layer. However, in the actual process, the multilayer transmission of information will always be weakened, so it is impossible to achieve exactly the same in the actual training. The training model parameters within the preset error range are considered to have achieved the training goal.

\subsection{DBN Model}

3.2.1. Overview of $D B N$. The deep trust network (DBN) is a deep structure composed of several restricted Boltzmann machines (RBM) and BP neural network. Its training process mainly includes the following two aspects: one is to use i-strong-m structure training to filter the relevant data feature information; the other is to connect each layer of RBM, establish BP neural network in the last layer, and use the output of RBM as BP neural network. Compared with the traditional neural network, DBN has the advantages of long training time, easy to fall into local optimum, and slow processing speed of big data.

3.2.2. RBM Algorithm. If $V_{\mathrm{i}}$ is the node value of visible layer, $H_{\mathrm{J}}$ is the node value of hidden layer, $A_{\mathrm{i}}$ and $B_{\mathrm{j}}$ are their offsets, and $W_{i j}$ is the weight between nodes, then

$$
P\left(h_{j}=1\right)=\frac{1}{1+\exp \left(-a_{j}-\sum_{i} v_{i} w_{i j}\right)} \text {. }
$$

Since the hidden layer and the visible layer can represent each other, there are

$$
P\left(v_{i}=1\right)=\frac{1}{1+\exp \left(-b_{i}-\sum_{j} h_{j} w_{j i}\right)} .
$$

Then train the CD (Contrastive Divergence) criterion of $\mathrm{RBM}$ and propose the update formula of weight vector $\theta(a, b, w)$ as

$$
\theta_{i+1}=\theta_{i}+u \frac{\partial \log _{p}(v, h)}{\partial \theta} \theta_{i} .
$$

\section{Discussion on Experimental Methods and Results}

\subsection{Experiment}

\subsubsection{Experimental Method}

(1) Fuzzy Theory. In this paper, the fuzzy theory is applied to the fault diagnosis of shunt capacitor, and people's long-term experience of fault diagnosis is expressed mathematically. On this basis, a kind of fuzzy fault diagnosis system of shunt capacitor is studied, which can accurately diagnose what kind of fault happened to the capacitor and its severity.

(2) Map Reduce-Based Distributed DBN. In this paper, the fault diagnosis and identification method of power capacitor based on Map Reduce distributed DBN is adopted, and the cloud computing-related technology is integrated into the deep learning. At the same time, some comparative analysis is made with SVM and DBN, so as to highlight the relevant advantages of MR-DBN algorithm in power capacitor fault diagnosis to a large extent.

\subsubsection{Correlative Design of Fuzzy Diagnosis Systems}

(1) Fuzzy Algorithm. There are four common algorithm models of fuzzy operator $m(\cdot,+)$. In order to consider the influence of all factors and keep all the information of single factor evaluation, the following algorithm is selected: 


$$
M(\cdot,+)=\sum_{i=1}^{m} x_{i} y_{i j}
$$

where $j=1,2, \ldots, \mathrm{n}$.

4.1.3. Overall Framework of the System. The overall frame structure is shown in Figure 1.

It can be seen from Figure 1 that the overall framework includes a main control operation module and four functional modules for data acquisition, and the four functional modules for data acquisition include signal analysis, feature extraction, fault diagnosis, and database.

\subsubsection{Map Reduce Model Process}

(1) Input. Read the relevant data in the distributed file system and divide the relevant data into certain data pieces. Each map function in the Map Reduce framework can allocate a data piece.

(2) Map. Data slicing is considered as a set of key-value pairs. According to the relevant program logic of the mapping function, the relevant key-value pairs allocated by Map Reduce framework are processed, and a new intermediate key-value pair is generated.

(3) Shuffle. In this stage, the intermediate key-value pair is transferred from the mapping node to the reduction node, and the same intermediate key-value pair is merged at the same time to form the intermediate key chain and key-value sorting.

(4) Reduce. Execute the reduce function.

(5) Output. Output the processing result of reduce function, and save the result in the specified distributed file system.

\subsubsection{Evaluation Index}

(1) The accuracy of power capacitor fault judgment is as follows:

$$
\text { accuracy }=\frac{\mathrm{a}}{\mathrm{b}} \times 100 \%
$$

(2) The error rate of power capacitor fault judgment is

$$
\text { False }_{i j}=\frac{c}{a} \times 100 \%
$$

\subsection{Analysis of Experimental Results}

4.2.1. Analysis of Fuzzy Diagnosis Results of Capacitor Fault Diagnosis. According to the symptoms of capacitor fault, combined with a large number of data related to the system in actual operation and the related data in parallel capacitors, according to the relevant membership method, carry out the relevant fuzzy diagnosis for the fault diagnosis of parallel capacitors; the specific situation is shown in Table 1.
It can be seen from Table 1 that the value of fuzzy diagnosis matrix reflects the close relationship between symptoms and fault causes, which is the quantification of $0-1$ fuzzy relationship between symptoms and faults in the process of fuzzy reasoning; in the initialization process of $\mathrm{R}$ value, there is inevitably subjective component, which may lead to wrong judgment. After a long time of operation, the performance and characteristic parameters of parallel capacitors will occur. In order to make the diagnosis conclusion more practical, it is necessary to modify $\mathrm{R}$ continuously during the operation of shunt capacitor.

4.2.2. Analysis of Fault Data Diagnosis Results Based on Fuzzy Theory. The diagnosis results of 60 groups of parallel capacitors using this system are shown in Table 2.

According to Table 2, the accuracy of the diagnosis results is $94 \%, 84 \%, 90 \%, 80 \%, 83 \%$, and $70 \%$, respectively; when the accuracy of the diagnosis results is greater than $90 \%$, it means that the reliability of the model diagnosis is very high; when the accuracy of the diagnosis results is greater than $75 \%$, it means that the reliability of the model diagnosis is relatively high; when the accuracy of the diagnosis results is less than $75 \%$, it means that the reliability of the model diagnosis is not to a large extent. In order to improve the reliability of model diagnosis, it is necessary to improve the fuzzy diagnosis matrix R.

4.2.3. Comparative Analysis of $M R-D B N$ and Other Methods in Detection Rate and Detection Time. The comparison of detection rate and detection time between MR-DBN and other methods is shown in Figure 2.

It can be seen from Figure 2 that the detection rates of three methods, SVM, DBN, and MR-DBN, are 96.43, 97.52, and 98.48, respectively. The RW detection rates of SVM, $\mathrm{DBN}$, and MR-DBN were 3.42, 2.45, and 2.31, respectively. The detection rates of SVM, DBN, and MR-DBN were 8.13, 7.52, and 7.54, respectively. The detection time of SVM, DBN, and MR-DBN was 20.31, 19.22, and 18.41, respectively. It can be seen that, among the three methods, the detection rate of MR-DBN is higher than that of the other two.

4.2.4. Analysis of Accuracy, Parameters, and MR-DBN Diagnosis Results of Different Algorithms. In order to verify the reliability and practicability of the fault diagnosis algorithm used in this paper, this paper compares the MR-DBN algorithm with the related support vector machine and DBN algorithm. The accuracy and parameters of different algorithms are shown in Figure 3, and the MR-DBN diagnosis results are shown in Figure 4.

It can be seen from Figures 3 and 4 that the diagnostic accuracy and misjudgment rate of DBN are, respectively, $96.33 \%$ and $3.90 \%$. The diagnosis accuracy and misjudgment rate of SVM are as follows: $96.40 \%$ and $3.83 \%$. The diagnostic accuracy and misjudgment rate of MR-DBN are as follows: $99.52 \%$ and $0.57 \%$. Therefore, compared with the other two methods, MR-DBN has the highest diagnostic accuracy and 


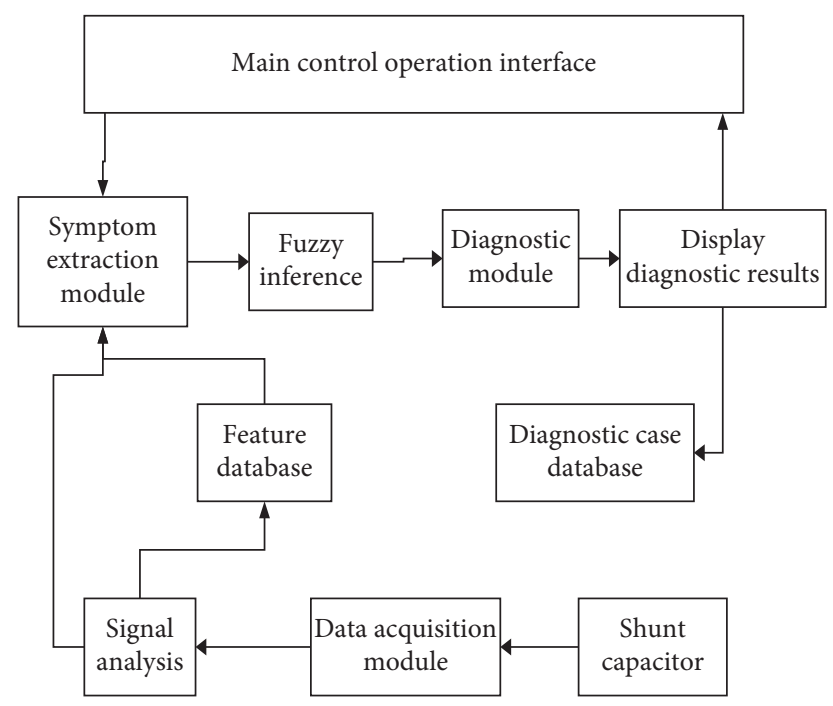

Figure 1: Overall structure of the system.

TABLE 1: Fault diagnosis matrix of shunt capacitor.

\begin{tabular}{lcccrr}
\hline \multirow{2}{*}{ Fault symptoms } & \multicolumn{4}{c}{ Cause of failure } \\
& $Y 1$ & $Y 2$ & $Y 3$ & $Y 4$ & 0.02 \\
\hline$X 1$ & 0.95 & 0.83 & 0.02 & 0.01 \\
$X 2$ & 0 & 0.01 & 0.02 & 0.86 \\
$X 3$ & 0.1 & 0 & 0.1 & 0.5 & 0.1 \\
\hline
\end{tabular}

TABLE 2: Summary of diagnosis results.

\begin{tabular}{lcc}
\hline Fault type & Group number & Diagnostic accuracy (\%) \\
\hline Shell deformation & 18 & 94 \\
Oil leakage & 24 & 84 \\
Overtemperature & 5 & 90 \\
Fuse blown & 6 & 80 \\
Splashing sparks and fire & 3 & 83 \\
Poor insulation & 4 & 70 \\
\hline
\end{tabular}

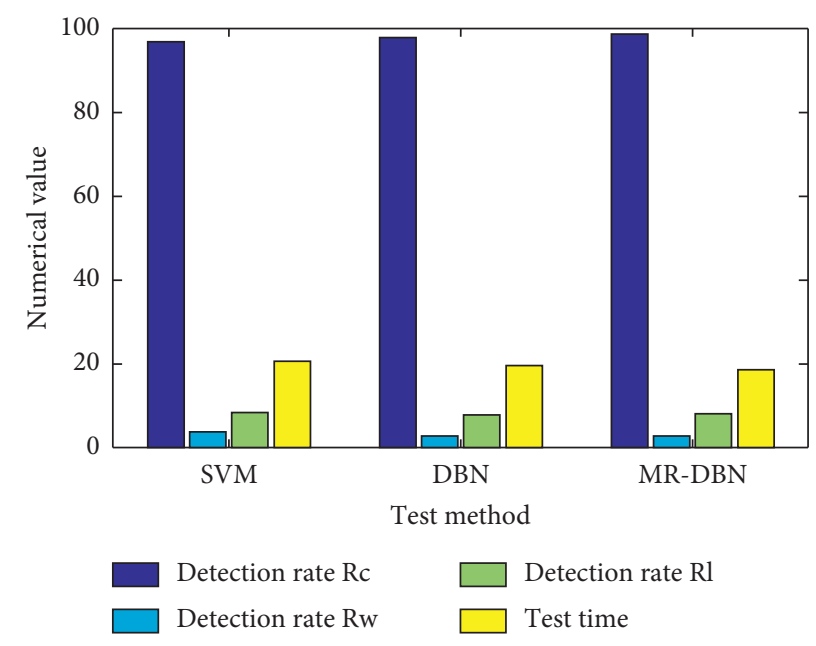

FIgURE 2: Comparison of detection rate and detection time between MR-DBN and other methods. 


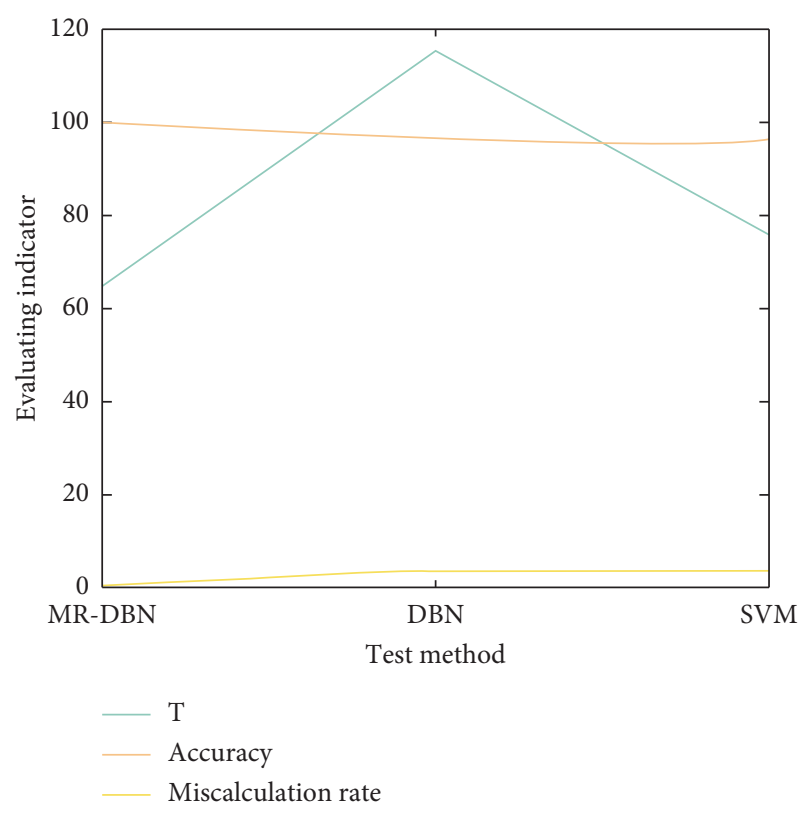

Figure 3: Accuracy and parameters of different algorithms.

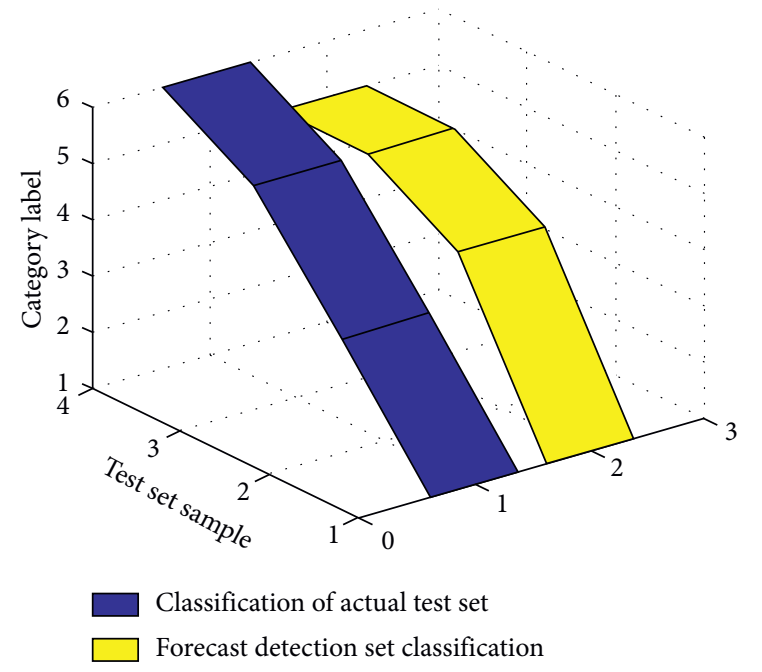

Figure 4: MR-DBN diagnosis results.

the lowest misjudgment rate; in the diagnosis results of MRDBN, the actual detection set is the same as the difference between the prediction detection sets is small.

4.2.5. Comparative Analysis of Diagnosis Results of Three Algorithms. The diagnosis results of the three algorithms are shown in Figure 5.

It can be seen from Figure 5 that the fault accuracy of the algorithm MR-DBN is $99.52 \%$, the number of misjudgments is 2 , the fault accuracy of $\mathrm{DBN}$ is $94.83 \%$, the number of misjudgments is 7, the fault accuracy of SVM is $91.43 \%$, and the number of misjudgments is 8 . By comparing the fault diagnosis accuracy of different methods and the number of wrong judgments, we can see that the MR-DBN algorithm has a high diagnosis rate and plays an important role in the

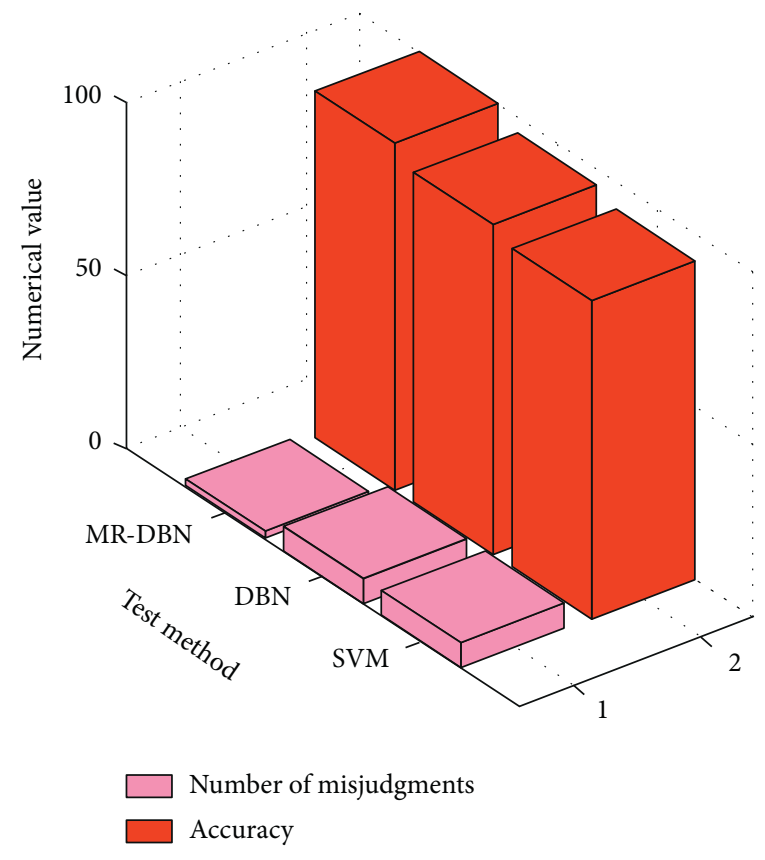

FIgURE 5: Comparison of diagnosis results.

related aspects of power capacitor fault diagnosis and identification. It not only improves the accuracy of power capacitor fault diagnosis and identification but also improves the stability of capacitor fault diagnosis and identification and provides a new method for the research and application of power container fault diagnosis and identification.

\section{Conclusions}

In this paper, the fuzzy diagnosis is applied to the fault diagnosis of shunt capacitor. Based on the operation experience of shunt capacitor, the fuzzy diagnosis matrix is established, the fuzzy algorithm and diagnosis principle are determined, and the fuzzy fault diagnosis system is designed. The results of this paper show that the fuzzy diagnosis method is effective in the fault diagnosis of parallel capacitors. In addition, in view of the related defects of traditional methods in the fault diagnosis of power capacitors, such as low accuracy, large error, and poor real-time performance, this paper also introduces the cloud computing technology into the deep learning and proposes a power capacitor fault diagnosis and solution based on map. In comparison with DBN and SVM, it is found that the diagnosis accuracy of the algorithm is relatively high.

The research of this paper shows that the accuracy of fuzzy diagnosis results is $94 \%, 84 \%, 90 \%, 80 \%, 83 \%$, and $70 \%$, respectively. When the accuracy of diagnosis results is greater than $90 \%$, it means that the reliability of model diagnosis is very high. When the accuracy of diagnosis results is greater than $75 \%$, it means that the reliability of model diagnosis is relatively high. When the accuracy of diagnosis results is less than $75 \%$, it means that the reliability of model diagnosis is not very good. In order to improve the reliability of model diagnosis, it is necessary to improve the 
fuzzy diagnosis matrix. Among the three methods, the diagnostic accuracy and misjudgment rate of DBN are $96.33 \%$ and $3.90 \%$, respectively, the diagnostic accuracy and misjudgment rate of SVM are $96.40 \%$ and $3.83 \%$, respectively, and the diagnostic accuracy and misjudgment rate of MRDBN are $99.52 \%$ and $0.57 \%$, respectively. Therefore, compared with the other two methods, the diagnostic accuracy and misjudgment rate of MR-DBN are the highest and the lowest, which to a large extent shows that the accuracy and misjudgment rate adopted in this paper are the lowest; the MR-DBN algorithm used in this paper has high accuracy in correlation diagnosis. It plays an important role in power capacitor fault diagnosis. It not only improves the correlation accuracy of capacitor fault diagnosis and identification but also provides a new method for related application research and analysis.

In the related power distribution system, capacitors play a very important role in the power system, but there are still some deficiencies in the corresponding fault analysis and research work. With the continuous improvement of the design level and manufacturing process of power equipment, the quality of capacitors will be further improved. At present, the integrated capacitor is slowly replacing the traditional capacitor bank, but new equipment and technology will also bring a series of problems. It is a very important but long-term task to analyze and deal with the related problems of capacitor.

\section{Data Availability}

No data were used to support this study.

\section{Conflicts of Interest}

The authors declare that they have no conflicts of interest.

\section{Acknowledgments}

This work was supported by the National Natural Science Foundation of China under Grant no. 61572343, the Research Fund Project of Shanxi Datong University under Grant no. 2017K11, and the 13th Five-Year Plan Project of Shanxi Education Science under Grant nos. GH-18044 and GH-19066.

\section{References}

[1] X. Wang, Y. Zhu, and Y. Wang, "Online identification method of power capacitor dielectric loss angle based on deep learning," Diangong Jishu Xuebao/Transactions of China Electrotechnical Society, vol. 32, no. 15, pp. 145-152, 2017.

[2] F. Chen, L. Z. Fu, and L. Zhen, "Thermal power generation fault diagnosis and prediction model based on deep learning and multimedia systems," Multimedia Tools \& Applications, vol. 78, no. 4, pp. 4673-4692, 2019.

[3] Y. Jian, X. Qing, and L. He, "Fault diagnosis of motor bearing based on deep learning," Advances in Mechanical Engineering, vol. 11, no. 9, 2019.

[4] G. Zhang, W. Bao, and X. Zhu, "A server consolidation method with integrated deep learning predictor in local storage based clouds," Concurrency, Practice and Experience, vol. 30, no. 23, pp. e4503.1-e4503.16, 2018.

[5] X. Hou and G. Zhao, "Resource scheduling and load balancing fusion algorithm with deep learning based on cloud computing," International Journal of Information Technology and Web Engineering, vol. 13, no. 3, pp. 54-72, 2018.

[6] W. Gao and Y. Zhu, "A cloud computing fault detection method based on deep learning," Journal of Computer \& Communications, vol. 05, no. 12, pp. 24-34, 2017.

[7] J. Tang, D. Sun, and S. Liu, "Enabling deep learning on IoT devices," Computer, vol. 50, no. 10, pp. 92-96, 2017.

[8] M. U. Yaseen, A. Anjum, and M. Farid, "Cloud-based video analytics using convolutional neural networks," Software Practice \& Experience, vol. 49, no. 4, pp. 565-583, 2019.

[9] J. Li, G. Luo, and N. Cheng, "An end-to-end load balancer based on deep learning for vehicular network traffic control," IEEE Internet of Things Journal, vol. 6, no. 1, pp. 953-966, 2019.

[10] S. Li, Y. Nie, and J. Li, "Condition monitoring and diagnosis of power equipment: review and prospective," High Voltage, vol. 2, no. 2, pp. 82-91, 2017.

[11] A. A. Elserougi, A. M. Massoud, and S. Ahmed, "Arrester-less DC fault current limiter based on pre-charged external capacitors for half-bridge modular multilevel converters," Generation, Transmission \& Distribution, IET, vol. 11, no. 1, pp. 93-101, 2017.

[12] J. Amini and M. Moallem, "A fault-diagnosis and fault-tolerant control scheme for flying capacitor multilevel inverters," Industrial Electronics, IEEE Transactions on, vol. 64, no. 3, pp. 1818-1826, 2017.

[13] J. Liu, Q. Li, and W. Chen, "A discrete hidden Markov model fault diagnosis strategy based on K-means clustering dedicated to PEM fuel cell systems of tramways," International Journal of Hydrogen Energy, vol. 43, no. 27, pp. 12428-12441, 2018.

[14] J. H. Jung, H. K. Ku, and Y. D. Son, "Open-switch fault diagnosis algorithm and tolerant control method of the threephase three-level NPC active rectifier," Energies, vol. 12, no. 13, p. 2495, 2019.

[15] H. Givi, E. Farjah, and T. Ghanbari, "Switch fault diagnosis and capacitor lifetime monitoring technique for DC-DC converters using a single sensor," Science, Measurement \& Technology, IET, vol. 10, no. 5, pp. 513-527, 2016.

[16] E. Farjah, H. Givi, and T. Ghanbari, "Application of an efficient rogowski coil sensor for switch fault diagnosis and capacitor ESR monitoring in nonisolated single-switch DC-DC converters," IEEE Transactions on Power Electronics, vol. 32, no. 2, pp. 1442-1456, 2017.

[17] F. Naseri, E. Farjah, and M. Allahbakhshi, "Online condition monitoring and fault detection of large supercapacitor banks in electric vehicle applications," Electrical Systems in Transportation, IET, vol. 7, no. 4, pp. 318-326, 2017.

[18] J. Hannonen, J. Honkanen, and J. P. Strm, "Capacitor aging detection in a DC-DC converter output stage," IEEE Transactions on Industry Applications, vol. 52, no. 4, pp. 3224-3233, 2016.

[19] W. Chen and A. M. Bazzi, "Logic-based methods for intelligent fault diagnosis and recovery in power electronics," IEEE Transactions on Power Electronics, vol. 32, no. 7, pp. 55735589, 2017.

[20] S. H. A. Niaki, S. M. Hosseini, and A. A. Abdoos, "Fault detection of HVDC cable in multi-terminal offshore wind farms using transient sheath voltage," Renewable Power Generation, IET, vol. 11, no. 13, pp. 1707-1713, 2017. 
[21] K. Yao, C. Cao, and S. Yang, "Noninvasive online condition monitoring of output capacitor's ESR and C for a flyback converter," Instrumentation \& Measurement IEEE Transactions on, vol. 66, no. 12, pp. 3190-3199, 2017.

[22] H. Jouybari-Moghaddam, T. S. Sidhu, and M. R. D. Zadeh, "Shunt capacitor banks online monitoring using a superimposed reactance method," Smart Grid IEEE Transactions on, vol. 9, no. 6, pp. 5554-5563, 2018.

[23] L. Liao, H. Gao, and Y. He, "Fault diagnosis of capacitance aging in DC link capacitors of voltage source inverters using evidence reasoning rule," Mathematical Problems in Engineering, vol. 2020, no. 9, pp. 1-12, 2020.

[24] K. Bi, Q. An, and J. Duan, "Fast diagnostic method of open circuit fault for modular multilevel DC/DC converter applied in energy storage system," IEEE Transactions on Power Electronics, vol. 32, no. 5, pp. 3292-3296, 2017.

[25] W. C. Santos, F. V. Lopes, and N. S. D. Brito, "High-impedance fault identification on distribution networks," Power Delivery IEEE Transactions on, vol. 32, no. 1, pp. 23-32, 2017. 